\title{
Etiologic Agents and Antifungal Susceptibility of Oral Candidosis from Romanian patients with HIV-infection or type 1 diabetes mellitus
}

\author{
BOGDAN MINEA ${ }^{1}$, VALENTIN NASTASA ${ }^{2}$, ANNA KOLECKA ${ }^{3}$, MAGDALENA MARES $^{4}$, \\ NARCISA MARANGOCI ${ }^{1}$, IRINA ROSCA ${ }^{1}$, MARIANA PINTEALA ${ }^{1}$, \\ MONICA HANCIANU ${ }^{5}$ and MIHAI MARES ${ }^{2 *}$
}

\author{
${ }^{1}$ Institute of Macromolecular Chemistry "Petru Poni”, Advanced Research Centre for Bionanoconjugates \\ and Biopolymers, Iasi, Romania \\ 2 "Ion Ionescu de la Brad" University, Laboratory of Antimicrobial Chemotherapy, Iasi, Romania \\ ${ }^{3}$ CBS Fungal Biodiversity Centre (CBS-KNAW), Yeast and Basidiomycete Research, Utrecht, Netherlands \\ ${ }^{4}$ Third School Health Centre, Department of Dentistry, Piatra Neamt, Romania \\ ${ }^{5}$ University of Medicine and Pharmacy “Gr. T. Popa”, Faculty of Pharmacy, Iasi, Romania
}

Submitted 11 March 2015, revised 15 April 2015, accepted 21 April 2015

\begin{abstract}
This is the first Romanian investigation of oral candidosis in patients suffering of HIV-infection or type 1 diabetes mellitus (T1DM). Candida albicans was the dominant species in both types of isolates: $\mathrm{n}=14(46.7 \%)$ in T1DM, $\mathrm{n}=60(69.8 \%)$ in HIV. The most frequent nonalbicans Candida spp. were Candida kefyr $(\mathrm{n}=6 ; 20 \%)$ in T1DM and Candida dubliniensis $(\mathrm{n}=8 ; 9.3 \%)$ in HIV. Resistance to fluconazole was detected only in the HIV non-albicans Candida group $(n=8 ; 9.3 \%)$. All isolates were susceptible to VOR. The experimental drug MXP had MIC values equal or close to the ones of VOR. Echinocandin resistance was more frequent than azole resistance.
\end{abstract}

Ke y w ord s: antifungal susceptibility, MXP-4509, oral candidosis, Romanian HIV and diabetes patients

The presence of oral Candida yeasts is considered a biomarker indicative of immune system impairment and, in immunodeficiency disorders, can be correlated with a progressive disease (Vargas and Joly, 2002). Oral candidosis (OC) is the most frequent type of yeast infection and occurs especially in denture wearers and individuals with severe conditions, such as HIV-infected patients, those under antibiotic or chemotherapy, organ transplantation recipients and patients with systemic diseases such as diabetes (Vergani et al., 2013).

HIV-infected patients are susceptible to opportunistic mycoses as cell-mediated immunity decays (Sangeorzan et al., 1994). Before the era of highly active antiretroviral therapy (HAART), oropharyngeal candidosis (OPC) occurred in as many as $90 \%$ of patients, at some point during the course of HIV infection (Lortholary et al., 2012). Since the initiation of HAART in 1996, there has been a decrease in the incidence of OPC (Leigh et al., 2004) while oropharingeal colonization varies from $44 \%$ to $62 \%$ (Lin et al., 2013a).
To the best of our knowledge, the present study is the first Romanian investigation providing data regarding the etiologic spectrum and the antifungal susceptibility profile of OC isolates from patients with either HIV-infection or diabetes.

The 116 clinical yeast isolates included in this study were collected in three tertiary hospitals from different regions of Romania (i.e. Iasi, Timisoara and Brasov), from patients with overt OC. Of these patients, 30 were suffering from type 1 (insulin-dependent) diabetes mellitus (T1DM), while the other 86 were HIV infected $\left(\mathrm{CD} 4+\mathrm{T}\right.$ lymphocytes count $\left.<500 / \mathrm{mm}^{3}\right)$. The final identification was performed using ID32C strips (bioMérieux, France). Isolates identified as Candida albicans or Candida dubliniensis were further verified with duplex PCR (Romeo and Criseo, 2011). The isolates for which the ID32C strips gave inconclusive results were sent to the CBS-KNAW Fungal Biodiversity Centre, Utrecht (The Netherlands), where they were identified by MALDI-TOF MS or the sequence analyses of the ITS

\footnotetext{
* Corresponding author: M. Mares, Laboratory of Antimicrobial Chemotherapy, "Ion Ionescu de la Brad" University, Iasi, Romania; e-mail: mycomedica@gmail.com
} 
(Internal Transcribed Spacer) and the D1/D2 domains of the LSU (Large SubUnit) of the ribosomal DNA, as previously reported (Kolecka et al., 2013).

In vitro susceptibility testing was performed following the EUCAST E. Def 7.1 guidelines (Subcommittee on Antifungal Susceptibility Testing (AFST) of the ESCMID European Committee for Antimicrobial Susceptibility Testing (EUCAST), 2008), for six antifungal agents: fluconazole (Sigma - St. Louis, USA), voriconazole (Pfizer Ltd. - Sandwich, UK), caspofungin (Merck \& Co, Inc.), micafungin (Astellas Pharma, Japan), anidulafungin (Pfizer, Inc.) and the MXP-4509 experimental compound ("Petru Poni" Institute of Macromolecular Chemistry - Iasi, Romania), which is a triazole based nanoconjugate with $\beta$-cyclodextrin as a carrier molecule (Marangoci et al., 2011). Two reference strains (C. albicans ATCC 90028 and Candida krusei ATCC 6258) were used for quality control. The interpretation of the MICs for the commercial antifungal agents was done according to the recent EUCAST document "Antifungal Agents. Breakpoint tables for interpretation of MICs", version 7.0 (Subcommittee on Antifungal Susceptibility Testing (AFST) of the European Committee for Antimicrobial Susceptibility Testing (EUCAST), 2014).

Specific statistical parameters (Mode, $\mathrm{MIC}_{50}$ and $\mathrm{MFC}_{50}$ - for $\mathrm{n} \geq 5, \mathrm{MIC}_{90}$ and $\mathrm{MFC}_{90}$ - for $\mathrm{n} \geq 10$ and Geometric Mean-for $n \geq 2$, where $n=$ the number of isolates) were calculated for each tested drug using Microsoft $^{\circledast}$ Excel $^{\circledast}$ (Dannaoui et al., 2008). Statistical analysis was performed using a fully functional trial version of GraphPad Prism version 6.04 for Windows, GraphPad Software, La Jolla California USA, www. graphpad.com. Two-tailed $P$-values were calculated and $P<0.05$ was considered significant. The level of significance was signalled in the text with one superscript asterisk $\left(^{*}\right)$ for $P \leq 0.05$ and two superscript asterisks $\left(^{* *}\right)$ for $P \leq 0.01$. To calculate the geometric means and run the statistical tests, right censored values (MIC > the maximum tested concentration) were treated as the next theoretical value i.e. " $>8 \mathrm{mg} / \mathrm{l}$ " was treated as "16 mg/l” (Dannaoui et al., 2008).

The overall species distribution and some of the calculated statistical parameters of the MICs are shown in Table I. Nine species were identified. In both types of chronic condition Candida albicans was the dominant species. Although it was surpassed by non-albicans Candida in the T1DM isolates, the statistical analysis revealed no significant differences in the distribution of species (C. albicans vs. non-albicans Candida) between the two categories. Cumulative antifungal susceptibility data $\left(\mathrm{MIC}_{50}, \mathrm{MIC}_{90}, \mathrm{MFC}_{50}, \mathrm{MFC}_{90}\right)$ along with susceptibility and resistance rates for $C$. albicans and the non-albicans Candida group are presented in Table II. All the T1DM isolates and all C. albicans HIV isolates were susceptible to FLC. Based mostly on the $2 \mathrm{mg} / \mathrm{l}$ non-specific BP for FLC, eight (30.77\%) non-albicans Candida HIV isolates can be considered resistant, i.e. four C. krusei isolates, two of Candida inconspicua and two of Candida norvegensis. There was also two intermediately susceptible Candida utilis isolate. All isolates were susceptible to VOR. Two C. albicans and two Candida tropicalis T1DM isolates were resistant to all echinocandins, but they were susceptible to azoles. The C. tropicalis isolates also had high MFC values for CAS and ANI. There were also two Candida lusitaniae T1DM isolates resistant to CAS. Within the HIV isolates there were four of $C$. albicans that were resistant to MCA and ANI but were susceptible to azoles. All the non-albicans Candida HIV isolates were susceptible to echinocandins.

The antifungals MICs for the reference strains used for quality control were: C. albicans ATCC 90028 (0.125-0.25 mg/l for FLC, $0.0156 \mathrm{mg} / \mathrm{l}$ for VOR, 0.0156-0.0312 mg/l for MXP, 0.0625-0.125 mg/l for CAS, $0.0156-0.0312$ for MCA, and $0.0156 \mathrm{mg} / \mathrm{l}$ for ANI); C. krusei ATCC 6258 (16-32 mg/l for FLC, $0.0625-0.125 \mathrm{mg} / \mathrm{l}$ for VOR, 0.0312-0.0625 mg/l for MXP, $0.0312-0.0625 \mathrm{mg} / \mathrm{l}$ for CAS, $0.0156-0.0312$ for MCA, and 0.0312-0.0625 mg/l for ANI).

Articles regarding species distribution and antifungal susceptibility of oral isolates from patients with diabetes are relatively scarce and, unlike our study, they investigate isolates resulted from colonisation and not from OC. Even fewer go as far as testing antifungal susceptibility. Despite reports of increased presence of nonalbicans Candida species, the most recent surveys from Brazil (Sanitá et al., 2013; Bremenkamp et al., 2011) or Western Europe (Manfredi et al., 2002; 2006) document isolation rates of $70 \%$ and higher for C. albicans. The proportion in our study, approximately $50 \%$, is more similar to reports from geographically closer areas such as Poland (Drozdowska and Drzewoski, 2008; Nawrot et al., 2006), Slovakia (Dorko et al., 2005) or Turkey (Kadir et al., 2002). Regarding the non-albicans species, most studies report the isolation of C. tropicalis, but also Candida glabrata and Candida parapsilosis; the latter two did not occur in our investigation. The number of isolates and also the geographical gradient are important reasons for these differences. The Turkish survey reports Candida kefyr, while an older British survey reports C. lusitaniae (Manfredi et al., 2002), species also reported by this study.

Our findings regarding FLC susceptibility are in agreement with the most recent Brazilian (Sanitá et al., 2013) and British-Italian (Manfredi et al., 2006) researches that found no FLC resistance. In contrast to the situation in Brazil, the Romanian isolates had a high rate of CAS resistance. Since there are no established BPs for CAS, we did use a non-specific BP of $0.25 \mathrm{mg} / \mathrm{l}$ which encompasses most of the already 
Table I

Species distribution and in vitro antifungal susceptibility in oral candidosis isolates

\begin{tabular}{|c|c|c|c|c|c|c|c|}
\hline \multirow{2}{*}{$\begin{array}{c}\text { Species (no. of isolates } \\
\% \text { of all isolates) }\end{array}$} & \multirow{2}{*}{$\begin{array}{l}\text { Com- } \\
\text { pound }\end{array}$} & \multicolumn{3}{|c|}{$\mathrm{MIC}(\mu \mathrm{g} / \mathrm{ml})$} & \multicolumn{3}{|c|}{ MFC $(\mu \mathrm{g} / \mathrm{ml})$} \\
\hline & & Range & Mode & $\mathrm{GM}^{1}$ & Range & Mode & GM \\
\hline \multirow{6}{*}{$\begin{array}{l}\text { T1DM isolates }(\mathbf{n}=\mathbf{3 0}) \\
\text { C. albicans } \\
(14-46.67 \% \text {, } \\
95 \% \mathrm{CI}=24.80-69.89 \%)\end{array}$} & FLC & $\leq 0.125-0.25$ & $\leq 0.125$ & 0.1682 & & & \\
\hline & VOR & $\mathrm{NA}^{1}$ & $\leq 0.0156$ & 0.1566 & & & \\
\hline & MXP & $\leq 0.0156-0.0625$ & $\leq 0.0156$ & 0.0210 & & & \\
\hline & CAS & $0.0312-0.5$ & 0.0312 & 0.0464 & $0.125-2.0$ & 0.25 & 0.4529 \\
\hline & MCA & $\leq 0.0156-0.25$ & $\leq 0.0156$ & 0.0283 & $0.0625-2.0$ & $0.125 ; 0.25$ & 0.2500 \\
\hline & ANI & $0.0312-0.25$ & 0.0312 & 0.0420 & $0.125-1.0$ & 0.125 & 0.2051 \\
\hline \multirow{6}{*}{$\begin{array}{l}\text { C. kefyr } \\
(6-20.00 \%)\end{array}$} & FLC & $0.25-0.5$ & 0.25 & 0.3150 & & & \\
\hline & VOR & NA & $\leq 0.0156$ & 0.0156 & & & \\
\hline & MXP & $\leq 0.0156-0.0312$ & $\leq 0.0156$ & 0.0197 & & & \\
\hline & CAS & NA & 0.0312 & 0.0312 & $0.0625-0.25$ & 0.25 & 0.1575 \\
\hline & MCA & $0.0312-0.0625$ & 0.0625 & 0.0496 & $0.125-0.5$ & NA & 0.2500 \\
\hline & ANI & $0.0625-0.25$ & NA & 0.1250 & $0.125-0.5$ & 0.5 & 0.3150 \\
\hline \multirow{6}{*}{$\begin{array}{l}\text { C. lusitaniae } \\
(6-20.00 \%)\end{array}$} & FLC & $\leq 0.125-0.5$ & $\leq 0.125$ & 0.1984 & & & \\
\hline & VOR & & $\leq 0.0156$ & 0.0156 & & & \\
\hline & MXP & $\leq 0.0156-0.0312$ & $\leq 0.0156$ & 0.0197 & & & \\
\hline & CAS & $0.0312-0.5$ & $\mathrm{NA}$ & 0.1249 & $0.5-1.0$ & 0.5 & 0.6300 \\
\hline & MCA & $0.0312-0.25$ & NA & 0.0787 & $0.125-0.5$ & 0.5 & 0.3150 \\
\hline & ANI & $0.0625-0.25$ & 0.0625 & 0.0992 & $0.25-1.0$ & 0.25 & 0.3969 \\
\hline \multirow{6}{*}{$\begin{array}{l}\text { C. tropicalis } \\
(4-13.33 \%)\end{array}$} & FLC & $\leq 0.125-1.0$ & NA & 0.3536 & & & \\
\hline & VOR & $\leq 0.0156-0.0312$ & NA & 0.0221 & & & \\
\hline & MXP & $\leq 0.0156-0.0625$ & NA & 0.0884 & & & \\
\hline & CAS & $0.0312-1.0$ & NA & 0.1766 & $0.25-16.0$ & $\mathrm{NA}$ & 2.0000 \\
\hline & MCA & $0.0625-1.0$ & NA & 0.2500 & $0.25-1.0$ & NA & 0.5000 \\
\hline & ANI & $0.0625-2.0$ & NA & 0.3536 & $1.0-8.0$ & NA & 2.8284 \\
\hline \multirow{6}{*}{$\begin{array}{l}\text { Non-albicans Candida } \\
(16-53.33 \% \text {, } \\
95 \% \text { CI }=30.11 \%-75.20 \%)\end{array}$} & FLC & $\leq 0.125-1.0$ & $\leq 0.125$ & 0.2726 & & & \\
\hline & VOR & $\leq 0.0156-0.0312$ & $\leq 0.0156$ & 0.0170 & & & \\
\hline & MXP & $\leq 0.0156-0.0625$ & $\leq 0.0156$ & 0.0286 & & & \\
\hline & CAS & $0.0312-1.0$ & 0.0312 & 0.0810 & $0.0625-16.0$ & 0.25 & 0.5000 \\
\hline & MCA & $0.0312-1.0$ & 0.0625 & 0.0884 & $0.125-1.0$ & & 0.3242 \\
\hline & ANI & $0.0625-2.0$ & 0.0625 & 0.1487 & $0.125-8.0$ & $0.25 ; 0.5 ; 1.0$ & 0.5946 \\
\hline \multirow[t]{6}{*}{ Overall } & FLC & $\leq 0.125-1.0$ & $\leq 0.125$ & 0.2176 & & & \\
\hline & VOR & $\leq 0.0156-0.0312$ & $\leq 0.0156$ & 0.0163 & & & \\
\hline & MXP & $\leq 0.0156-0.0625$ & $\leq 0.0156$ & 0.0248 & & & \\
\hline & CAS & $0.0312-1.0$ & 0.0312 & 0.0624 & $0.0625-16.0$ & 0.25 & 0.4774 \\
\hline & MCA & $\leq 0.0156-1.0$ & $\leq 0.0156 ; 0.0625$ & 0.0519 & $0.0625-2.0$ & $0.125 ; 0.25 ; 0.5$ & 0.2872 \\
\hline & ANI & $0.0312-2.0$ & 0.0312 & 0.0824 & $0.0625-8.0$ & 0.125 & 0.3618 \\
\hline \multirow{6}{*}{$\begin{array}{l}\text { HIV isolates }(\mathbf{n}=\mathbf{8 6}) \\
\text { C. albicans } \\
(60-69.77 \% \text {, } \\
95 \% \mathrm{CI}=54.80 \%-81.49 \%)\end{array}$} & FLC & $\leq 0.125-0.5$ & 0.5 & 0.2872 & & & \\
\hline & VOR & NA & $\leq 0.0156$ & 0.0156 & & & \\
\hline & MXP & NA & $\leq 0.0156$ & 0.0156 & & & \\
\hline & CAS & $\leq 0.0156-0.0312$ & 0.0312 & 0.0291 & $0.0625-2.0$ & 0.0625 & 0.1984 \\
\hline & MCA & $\leq 0.0156-0.0312$ & $\leq 0.0156$ & 0.0163 & $0.0312-2.0$ & 0.0625 & 0.1575 \\
\hline & ANI & $\leq 0.0156-0.0625$ & 0.0312 & 0.0312 & $0.0625-2.0$ & 0.0625 & 0.1469 \\
\hline \multirow{6}{*}{$\begin{array}{l}\text { C. dubliniensis } \\
(8-9.30 \%)\end{array}$} & FLC & $0.25-0.5$ & 0.25 & 0.2973 & & & \\
\hline & VOR & NA & $\leq 0.0156$ & 0.0156 & & & \\
\hline & MXP & NA & $\leq 0.0156$ & 0.0156 & & & \\
\hline & CAS & NA & 0.0312 & 0.0312 & $0.5-1.0$ & 1.0 & 0.8409 \\
\hline & MCA & $0.0312-0.0625$ & $0.0312 ; 0.0625$ & 0.0442 & $1.0-4.0$ & 1.0 & 1.4142 \\
\hline & ANI & $0.0625-0.125$ & 0.125 & 0.1051 & $0.125-1.0$ & 0.5 & 0.4204 \\
\hline \multirow{6}{*}{$\begin{array}{l}\text { C. kefyr } \\
(4-4.65 \%)\end{array}$} & FLC & NA & 0.5 & 0.5000 & & & \\
\hline & VOR & NA & $\leq 0.0156$ & 0.0156 & & & \\
\hline & MXP & NA & $\leq 0.0156$ & 0.0156 & & & \\
\hline & CAS & NA & 0.0312 & 0.0312 & $0.0312-0.0625$ & $\mathrm{NA}$ & 0.0442 \\
\hline & MCA & NA & 0.0625 & 0.0625 & $0.125-0.25$ & NA & 0.1768 \\
\hline & ANI & NA & 0.125 & 0.1250 & $0.25-0.5$ & NA & 0.3536 \\
\hline
\end{tabular}


Table I. Continued.

\begin{tabular}{|c|c|c|c|c|c|c|c|}
\hline \multirow{2}{*}{$\begin{array}{l}\text { Species (no. of isolates } \\
\% \text { of all isolates) }\end{array}$} & \multirow{2}{*}{$\begin{array}{l}\text { Com- } \\
\text { pound }\end{array}$} & \multicolumn{3}{|c|}{$\mathrm{MIC}(\mu \mathrm{g} / \mathrm{ml})$} & \multicolumn{3}{|c|}{$\operatorname{MFC}(\mu \mathrm{g} / \mathrm{ml})$} \\
\hline & & Range & Mode & $\mathrm{GM}^{1}$ & Range & Mode & GM \\
\hline \multirow[t]{2}{*}{$\begin{array}{l}\text { C. krusei } \\
(4-4.65 \%)\end{array}$} & $\begin{array}{l}\text { FLC } \\
\text { VOR } \\
\text { MXP }\end{array}$ & $\begin{array}{l}32.0-64.0 \\
0.25-0.5 \\
0.25-0.5\end{array}$ & $\begin{array}{l}\text { NA } \\
\text { NA } \\
\text { NA }\end{array}$ & $\begin{array}{c}45.2548 \\
0.3536 \\
0.3536\end{array}$ & & & \\
\hline & $\begin{array}{l}\text { CAS } \\
\text { MCA } \\
\text { ANI }\end{array}$ & $\begin{array}{l}\text { NA } \\
0.0625-0.125 \\
\text { NA }\end{array}$ & $\begin{array}{c}0.125 \\
\mathrm{NA} \\
0.0625\end{array}$ & $\begin{array}{l}0.1250 \\
0.0884 \\
0.0625\end{array}$ & $\begin{array}{l}0.125-0.25 \\
0.125-0.25 \\
\text { NA }\end{array}$ & $\begin{array}{l}\text { NA } \\
\text { NA } \\
0.125\end{array}$ & $\begin{array}{l}0.1768 \\
0.1768 \\
0.1250\end{array}$ \\
\hline \multirow[t]{2}{*}{$\begin{array}{l}\text { C. tropicalis } \\
(4-4.65 \%)\end{array}$} & $\begin{array}{l}\text { FLC } \\
\text { VOR } \\
\text { MXP }\end{array}$ & $\begin{array}{l}0.25-0.5 \\
\text { NA } \\
0.0312-0.0625\end{array}$ & $\begin{array}{c}\text { NA } \\
0.0312 \\
\text { NA }\end{array}$ & $\begin{array}{l}0.3536 \\
0.0312 \\
0.0442\end{array}$ & & & \\
\hline & $\begin{array}{l}\text { CAS } \\
\text { MCA } \\
\text { ANI }\end{array}$ & $\begin{array}{l}\text { NA } \\
\text { NA } \\
\text { NA }\end{array}$ & $\begin{array}{l}0.0312 \\
0.0312 \\
0.0625\end{array}$ & $\begin{array}{l}0.0312 \\
0.0312 \\
0.0625\end{array}$ & $\begin{array}{l}0.125-2.0 \\
\text { NA } \\
0.25-1.0\end{array}$ & $\begin{array}{l}\text { NA } \\
1.0 \\
\text { NA }\end{array}$ & $\begin{array}{l}0.5000 \\
1.0000 \\
0.5000\end{array}$ \\
\hline \multirow[t]{2}{*}{$\begin{array}{l}\text { C. inconspicua } \\
(2-2.33 \%)\end{array}$} & $\begin{array}{l}\text { FLC } \\
\text { VOR } \\
\text { MXP }\end{array}$ & $\begin{array}{l}\text { NA } \\
\text { NA } \\
\text { NA }\end{array}$ & $\begin{array}{c}32.0 \\
0.25 \\
0.125\end{array}$ & $\begin{array}{c}32 \\
0.25 \\
0.125\end{array}$ & & & \\
\hline & $\begin{array}{l}\text { CAS } \\
\text { MCA } \\
\text { ANI }\end{array}$ & $\begin{array}{l}\text { NA } \\
\text { NA } \\
\text { NA }\end{array}$ & $\begin{array}{c}0.125 \\
0.0312 \\
0.0625\end{array}$ & $\begin{array}{c}0.125 \\
0.0312 \\
0.0625\end{array}$ & $\begin{array}{l}\mathrm{NA} \\
\mathrm{NA} \\
\mathrm{NA}\end{array}$ & $\begin{array}{l}0.125 \\
0.0625 \\
0.125\end{array}$ & $\begin{array}{l}\text { NA } \\
\text { NA } \\
\text { NA }\end{array}$ \\
\hline \multirow[t]{2}{*}{$\begin{array}{l}\text { C. norvegensis } \\
(2-2.33 \%)\end{array}$} & $\begin{array}{l}\text { FLC } \\
\text { VOR } \\
\text { MXP }\end{array}$ & $\begin{array}{l}\text { NA } \\
\text { NA } \\
\text { NA }\end{array}$ & $\begin{array}{c}16.0 \\
0.0312 \\
0.0312\end{array}$ & $\begin{array}{c}16 \\
0.0312 \\
0.0312\end{array}$ & & & \\
\hline & $\begin{array}{l}\text { CAS } \\
\text { MCA } \\
\text { ANI }\end{array}$ & $\begin{array}{l}\text { NA } \\
\text { NA } \\
\text { NA }\end{array}$ & $\begin{array}{l}0.0625 \\
0.0312 \\
0.0312\end{array}$ & $\begin{array}{l}0.0625 \\
0.0312 \\
0.0312\end{array}$ & $\begin{array}{l}\mathrm{NA} \\
\mathrm{NA} \\
\mathrm{NA}\end{array}$ & $\begin{array}{l}0.25 \\
0.125 \\
0.125\end{array}$ & $\begin{array}{l}\text { NA } \\
\text { NA } \\
\text { NA }\end{array}$ \\
\hline \multirow[t]{2}{*}{$\begin{array}{l}\text { C. utilis } \\
(2-2.33 \%)\end{array}$} & $\begin{array}{l}\text { FLC } \\
\text { VOR } \\
\text { MXP }\end{array}$ & $\begin{array}{l}\text { NA } \\
\text { NA } \\
\text { NA }\end{array}$ & $\begin{array}{c}4.0 \\
0.125 \\
0.0625\end{array}$ & $\begin{array}{c}4 \\
0.125 \\
0.0625\end{array}$ & & & \\
\hline & $\begin{array}{l}\text { CAS } \\
\text { MCA } \\
\text { ANI }\end{array}$ & $\begin{array}{l}\text { NA } \\
\text { NA } \\
\text { NA }\end{array}$ & $\begin{array}{l}0.0312 \\
0.0312 \\
0.0312\end{array}$ & $\begin{array}{l}0.0312 \\
0.0312 \\
0.0312\end{array}$ & $\begin{array}{l}\mathrm{NA} \\
\mathrm{NA} \\
\mathrm{NA}\end{array}$ & $\begin{array}{l}0.0312 \\
0.0625 \\
0.0312\end{array}$ & $\begin{array}{l}\text { NA } \\
\text { NA } \\
\text { NA }\end{array}$ \\
\hline \multirow[t]{2}{*}{$\begin{array}{l}\text { Non-albicans Candida } \\
(26-30.23 \% \text {, } \\
95 \% \text { CI }=18.51 \%-45.20 \%)\end{array}$} & $\begin{array}{l}\text { FLC } \\
\text { VOR } \\
\text { MXP }\end{array}$ & $\begin{array}{l}0.25-64.0 \\
\leq 0.0156-0.5 \\
\leq 0.0156-0.5\end{array}$ & $\begin{array}{l}0.25 ; 0.5 \\
\leq 0.0156 \\
\leq 0.0156\end{array}$ & $\begin{array}{l}1.7044 \\
0.0430 \\
0.0408\end{array}$ & & & \\
\hline & $\begin{array}{l}\text { CAS } \\
\text { MCA } \\
\text { ANI }\end{array}$ & $\begin{array}{l}0.0312-0.125 \\
0.0312-0.125 \\
0.0312-0.125\end{array}$ & $\begin{array}{l}0.0312 \\
0.0312 \\
0.0625\end{array}$ & $\begin{array}{l}0.0453 \\
0.0453 \\
0.0733\end{array}$ & $\begin{array}{l}0.0312-2.0 \\
0.0625-4.0 \\
0.0312-1.0\end{array}$ & $\begin{array}{l}0.125 ; 1.0 \\
1.0 \\
0.125\end{array}$ & $\begin{array}{l}0.2370 \\
0.3631 \\
0.2370\end{array}$ \\
\hline \multirow[t]{2}{*}{ Overall } & $\begin{array}{l}\text { FLC } \\
\text { VOR } \\
\text { MXP }\end{array}$ & $\begin{array}{l}\leq 0.125-64.0 \\
\leq 0.0156-0.5 \\
\leq 0.0156-0.5\end{array}$ & $\begin{array}{c}0.5 \\
\leq 0.0156 \\
\leq 0.0156\end{array}$ & $\begin{array}{l}0.4920 \\
0.0212 \\
0.0209\end{array}$ & & & \\
\hline & $\begin{array}{l}\text { CAS } \\
\text { MCA } \\
\text { ANI }\end{array}$ & $\begin{array}{l}\leq 0.0156-0.125 \\
\leq 0.0156-0.125 \\
\leq 0.0156-0.125\end{array}$ & $\begin{array}{l}0.0312 \\
0.0156 \\
0.0312\end{array}$ & $\begin{array}{l}0.0333 \\
0.0222 \\
0.0404\end{array}$ & $\begin{array}{l}0.0312-2.0 \\
0.0312-4.0 \\
0.0312-2.0\end{array}$ & $\begin{array}{l}0.125 \\
0.0625 \\
0.125\end{array}$ & $\begin{array}{l}0.2094 \\
0.2027 \\
0.1698\end{array}$ \\
\hline
\end{tabular}

${ }^{1}$ GM-Geometric Mean; ${ }^{2}$ NA-Not Applicable

established echinocandin BPs. This situation requires further research, especially considering the findings of a recent study that echinocandins would be a safer choice for diabetes patients since they do not seem to be affected by glucose, which appears to significantly lower the antifungal activity of azoles and polyenes (Mandal et al., 2014).

Studies that investigate isolates from HIV patients are more abundant, but similarly to those targeting dia- betes, more of them focus on the asymptomatic carriage of yeasts in the oral cavities. Nevertheless, oral colonisation of HIV-infected patients in conjunction to low counts of CD4 cells are strong premises for subsequent development of OPC (Fong et al., 1997). The same increase of prevalence for the non-albicans species is documented for HIV patients and, equally, C. albicans remains the dominant species. C. dubliniensis, C. glabrata, and C.tropicalis are considered as 
Table II

Cumulative antifungal susceptibility data and resistance $(\mathrm{R})$ rates of oral candidosis isolates

\begin{tabular}{|c|c|c|c|c|c|c|c|c|c|}
\hline \multirow{3}{*}{ Species } & \multicolumn{4}{|c|}{ T1DM } & \multicolumn{5}{|c|}{ HIV } \\
\hline & \multirow{2}{*}{$\begin{array}{l}\text { Com- } \\
\text { pound }\end{array}$} & \multirow{2}{*}{$\begin{array}{l}\mathrm{MIC}_{50} \\
(\mu \mathrm{g} / \mathrm{ml})\end{array}$} & \multirow{2}{*}{$\begin{array}{l}\mathrm{MFC}_{50} \\
(\mu \mathrm{g} / \mathrm{ml})\end{array}$} & \multirow{2}{*}{$\mathrm{R}(\mathrm{n}-\%)$} & \multicolumn{2}{|c|}{ MIC $(\mu \mathrm{g} / \mathrm{ml})$} & \multicolumn{2}{|c|}{$\operatorname{MFC}(\mu \mathrm{g} / \mathrm{ml})$} & \multirow{2}{*}{$\mathrm{R}(\mathrm{n}-\%)$} \\
\hline & & & & & $\mathrm{MIC}_{50}$ & $\mathrm{MIC}_{90}$ & $\mathrm{MFC}_{50}$ & $\mathrm{MFC}_{90}$ & \\
\hline \multirow{6}{*}{$\begin{array}{l}\text { Candida } \\
\text { albicans }\end{array}$} & FLC & $\leq 0.125$ & & $0-0.0 \%$ & 0.25 & 0.5 & & & $0-0.0 \%$ \\
\hline & VOR & $\leq 0.0156$ & & $0-0.0 \%$ & $\leq 0.0156$ & $\leq 0.0156$ & & & $0-0.0 \%$ \\
\hline & MXP & $\leq 0.0156$ & & NA & $\leq 0.0156$ & $\leq 0.0156$ & & & NA \\
\hline & CAS & 0.0312 & 0.25 & $2-14.3 \%$ & 0.0312 & 0.0312 & 0.125 & 1.0 & $0-0.0 \%$ \\
\hline & MCA & $\leq 0.0156$ & 0.25 & $4-28.6 \%$ & $\leq 0.0156$ & $\leq 0.0156$ & 0.0625 & 2.0 & $4-6.7 \%$ \\
\hline & ANI & 0.0312 & 0.125 & $2-14.3 \%$ & 0.0312 & 0.0312 & 0.125 & 0.5 & $4-6.7 \%$ \\
\hline \multirow{6}{*}{$\begin{array}{l}\text { Non-albicans } \\
\text { Candida }\end{array}$} & FLC & 0.25 & & $0-0.0 \%$ & 0.5 & 32.0 & & & $8-30.8 \%$ \\
\hline & VOR & $\leq 0.0156$ & & $0-0.0 \%$ & 0.0312 & 0.25 & & & $0-0.0 \%$ \\
\hline & MXP & $\leq 0.0156$ & & NA & 0.0312 & 0.25 & & & NA \\
\hline & CAS & 0.0312 & 0.25 & $4-25.0 \%$ & 0.0312 & 0.125 & 0.25 & 1.0 & $0-0.0 \%$ \\
\hline & MCA & 0.0625 & 0.25 & $2-12.5 \%$ & 0.0312 & 0.0625 & 0.25 & 1.0 & $0-0.0 \%$ \\
\hline & ANI & 0.0625 & 0.5 & $2-12.5 \%$ & 0.0625 & 0.125 & 0.25 & 1.0 & $0-0.0 \%$ \\
\hline \multirow[t]{6}{*}{ Overall } & FLC & 0.25 & & $0-0.0 \%$ & 0.5 & 4.0 & & & $8-9.3 \%$ \\
\hline & VOR & $\leq 0.0156$ & & $0-0.0 \%$ & $\leq 0.0156$ & 0.0312 & & & $0-0.0 \%$ \\
\hline & MXP & $\leq 0.0156$ & & NA & $\leq 0.0156$ & 0.0625 & & & NA \\
\hline & $\mathrm{CAS}$ & 0.0312 & 0.25 & $6-20.0 \%$ & 0.0312 & 0.0312 & 0.125 & 1.0 & 0.0 \\
\hline & MCA & 0.0625 & 0.25 & $6-20.0 \%$ & $\leq 0.0156$ & 0.0625 & 0.125 & 2.0 & $4-4.7 \%$ \\
\hline & ANI & 0.0625 & 0.25 & $4-13.3 \%$ & 0.0312 & 0.125 & 0.125 & 0.5 & $4-4.7 \%$ \\
\hline
\end{tabular}

emerging pathogens (Lin et al., 2013; Drozdowska and Drzewoski, 2008; Binolfi et al., 2005).

Regarding C.albicans proportion within the HIV isolates, values similar to the one in this study (70\%) have been reported for Taiwan (Ho et al., 2014), Cameroun (dos Santos Abrantes et al., 2014), USA (Merenstein et al., 2013), Spain (Ramírez et al., 2006) or Turkey (Erköse and Erturan, 2007). Percentages can go as high as $90 \%$ in India (Maurya et al., 2013), Italy (Giammanco et al., 2002) or UK (Cartledge et al., 1999), 83\% in South Africa (dos Santos Abrantes et al., 2014), 79\% in Serbia (Mitrovic et al., 1996), or can go as low as 62\% in Turkey (Erköse and Erturan, 2007) or Brazil (Costa et al., 2006). Again, C. glabrata is missing from the isolates in our non-albicans Candida group.

Reported levels of FLC resistance vary widely from $0.9 \%$ in Taiwan (Ho et al., 2014) and 3.4\% in China (Li et al., 2013) to about $50 \%$ in South Africa and Cameroun (dos Santos Abrantes etal., 2014) for C. albicans. The differences can have a few possible causes such as street availability of antifungals, without prescription (dos Santos Abrantes et al., 2014), or the different susceptibility testing methods used in each investigation. In our study, FLC resistance was present only in the non-albicans Candida group, in agreement with the above mentioned sources which found higher resistance rates for this group by up to $13 \%$ (Li et al., 2013).

All the Romanian isolates were susceptible to VOR, a situation similar to that in Taiwan (Ho et al., 2014).
Some resistance was found in China-3\% for C. albicans and $14.5 \%$ for non-albicans Candida (Li et al., 2013) and very high values, were reported for C.albicans from South Africa and Cameroun (dos Santos Abrantes et al., 2014). This study also signals the occurrence of resistance to echinocandins that other investigations did not report. Although these antifungals are not the first choice in treating patients with OC, they can be an effective alternative if topical or systemic azoles have definitely failed (Lortholary et al., 2012).

Our study confirms a few worldwide reported tendencies such as the increasing prevalence and lower antifungal susceptibility of non-albicans Candida species, and C. dubliniensis as an emerging oral pathogen in HIV patients. It also supports the status of FLC as the first option for treatment, but not advisable for prophylaxis, and VOR as a viable second line of defence.

As a triazole based antifungal, MXP-4509 inhibits the ergosterol biosynthesis, similar to FLC and VOR. The experimental drug had a good antifungal activity with MIC values similar to those of VOR. Further, in vivo studies are warranted.

In conclusion, strict oral hygiene and adherence to specific treatment are the best prophylactic approaches to prevent OC in both chronic conditions, while FLC is recommended only as a first line of defense after the occurrence of the infection. As a second line of defense, in case of FLC therapeutic failure, echinocandins are a viable option for HIV patients. In the case 
of diabetes patients, however, the risk of azole crossresistance should be evaluated first; for patients without prior exposure to azoles, VOR may be a better option.

\section{Acknowledgements}

This publication benefited from the financial support of the project "Programme of excellency in the multidisciplinary doctoral and post-doctoral research of chronic diseases", contract no. POSDRU/159/1.5/S/133377, beneficiary "Gr. T. Popa” University of Medicine and Pharmacy of Iasi, project co-financed from the European Social Fund through the Sectoral Operational Programme Human Resources Development (SOP HRD) 2007-2013.

\section{Literature}

Binolfi A., M.S. Biasoli, A.G. Luque, M.E. Tosello and H.M. Magaró. 2005. High prevalence of oral colonization by Candida dubliniensis in HIV-positive patients in Argentina. Med. Mycol. 43(5): 431-437.

Bremenkamp R.M., A.R. Caris, A.O.C. Jorge, G.N. Back-Brito, A.J. Mota, I. Balducci, F.L. Brighenti and C.Y. Koga-Ito. 2011. Prevalence and antifungal resistance profile of Candida spp. oral isolates from patients with type 1 and 2 diabetes mellitus. Arch. Oral Biol. 56(6): 549-555.

Cartledge J.D., J. Midgley and B.G. Gazzard. 1999. Non-albicans oral candidosis in HIV-positive patients. J. Antimicrob. Chemother. 43(3): 419-422.

Costa C.R., J.A. de Lemos, X.S. Passos, C.R. de Araújo, A.J. Cohen, L.K.H.E. Souza and M. do R.R. Silva. 2006. Species distribution and antifungal susceptibility profile of oral Candida isolates from HIV-infected patients in the antiretroviral therapy era. Mycopathologia 162(1): 45-50.

Dannaoui E., O. Lortholary, D. Raoux, M.E. Bougnoux, G. Galeazzi, C. Lawrence, D. Moissenet, I. Poilane, D. Hoinard and F. Dromer. 2008. Comparative in vitro activities of caspofungin and micafungin, determined using the method of the European Committee on antimicrobial susceptibility testing, against yeast isolates obtained in France in 2005-2006. Antimicrob. Agents Chemother. 52 (2): 778-781.

Dorko E., Z. Baranová, A. Jenča, P. Kizek, E. Pilipčinec and L. Tkáčiková. 2005. Diabetes mellitus and candidiases. Folia Microbiol. (Praha) 50(3): 255-261.

Dos Santos Abrantes P.M., C.P. McArthur and C.W.J. Africa. 2014. Multi-drug resistant oral Candida species isolated from HIVpositive patients in South Africa and Cameroon. Diagn. Microbiol. Infect. Dis. 79(2): 222-227.

Drozdowska A. and J. Drzewoski. 2008. Mycoses in diabetesdifficult diagnostic and therapeutic problem. Review of literature. Diabetol. Doświadczalna Klin. 8(1): 1-11.

Erköse G. and Z. Erturan. 2007. Oral Candida colonization of human immunodeficiency virus infected subjects in Turkey and its relation with viral load and CD4+ T-lymphocyte count. Mycoses 50(6): 485-490.

Fong I.W., M. Laurel and A. Burford-Mason. 1997. Asymptomatic oral carriage of Candida albicans in patients with HIV infection. Clin. Investig. Med. Médecine Clin. Exp. 20(2): 85-93.

Giammanco G.M., G. Pizzo, S. Pecorella, S. Distefano, V. Pecoraro and M.E. Milici. 2002. Identification of Candida dubliniensis among oral yeast isolates from an Italian population of human immunodeficiency virus-infected (HIV+) subjects. Oral Microbiol. Immunol. 17(2): 89-94.
Ho M.-W., Y.-L. Yang, C.-C. Lin, C.-Y. Chi, H.-T. Chen, P.-C. Lin, L.-Y. Hsieh, C.-H. Chou, W.-L. Chu, C.-P. Wu and others. 2014. Yeast oropharyngeal colonization in human immunodeficiency virus-infected patients in central Taiwan. Mycopathologia 177(5-6): 309-317.

Kadir T., R. Pisiriciler, S. Akyüz, A. Yarat, N. Emekli and A. Ipbüker. 2002. Mycological and cytological examination of oral candidal carriage in diabetic patients and non-diabetic control subjects: thorough analysis of local aetiologic and systemic factors. J. Oral Rehabil. 29(5): 452-457.

Kolecka A., K. Khayhan, M. Groenewald, B. Theelen, M. Arabatzis, A. Velegraki, M. Kostrzewa, M. Mares, S.J. Taj-Aldeen and T. Boekhout. 2013. Identification of medically relevant species of arthroconidial yeasts by use of matrix-assisted laser desorption ionization-time of flight mass spectrometry. J. Clin. Microbiol. 51(8): 2491-2500

Leigh J.E., K. Shetty and P.L. Fidel. 2004. Oral opportunistic infections in HIV-positive individuals: review and role of mucosal immunity. AIDS Patient Care STDs 18(8): 443-56.

Lin J.-N., C.-C. Lin, C.-H. Lai, Y.-L. Yang, H.-T. Chen, H.-C. Weng, L.-Y. Hsieh, Y.-C. Kuo, T.-L. Lauderdale, F.-C. Tseng and others. 2013. Predisposing factors for oropharyngeal colonization of yeasts in human immunodeficiency virus-infected patients: A prospective cross-sectional study. J. Microbiol. Immunol. Infect. 46(2): 129-135. Li Y.-Y., W.-Y. Chen, X. Li, H.-B. Li, H.-Q. Li, L. Wang, L. He, X.-P. Yang, X.-C. Wang, Y.-L. Huang and others. 2013. Asymptomatic oral yeast carriage and antifungal susceptibility profile of HIV-infected patients in Kunming, Yunnan Province of China. BMC Infect. Dis. 13(1): 46.

Lortholary O., G. Petrikkos, M. Akova, M.C. Arendrup, S. Arikan-Akdagli, M. Bassetti, J. Bille, T. Calandra, E. Castagnola, O.A. Cornely and others. 2012. ESCMID ${ }^{\star}$ guideline for the diagnosis and management of Candida diseases 2012: patients with HIV infection or AIDS. Clin. Microbiol. Infect. 18(Suppl. 7): 68-77.

Mandal S.M., D. Mahata, L. Migliolo, A. Parekh, P.S. Addy, M. Mandal and A. Basak. 2014. Glucose directly promotes antifungal resistance in the fungal pathogen, Candida spp. J. Biol. Chem. 289(37): 25468-25473.

Manfredi M., M.J. McCullough, Z.M. Al-Karaawi, S.J. Hurel and S.R. Porter. 2002. The isolation, identification and molecular analysis of Candida spp. isolated from the oral cavities of patients with diabetes mellitus. Oral Microbiol. Immunol. 17(3): 181-185.

Manfredi M., M.J. McCullough, L. Polonelli, S. Conti, Z.M. AlKaraawi, P. Vescovi and S.R. Porter. 2006. In vitro antifungal susceptibility to six antifungal agents of 229 Candida isolates from patients with diabetes mellitus. Oral Microbiol. Immunol. 21(3): 177-182.

Marangoci N., M. Mares, M. Silion, A. Fifere, C. Varganici, A. Nicolescu, C. Deleanu, A. Coroaba, M. Pinteala and B.C. Simionescu. 2011. Inclusion complex of a new propiconazole derivative with $\beta$-cyclodextrin: NMR, ESI-MS and preliminary pharmacological studies. Results Pharma Sci. 1(1): 27-37.

Maurya V., A. Srivastava, J. Mishra, R. Gaind, R.S.K. Marak, A.K. Tripathi, M. Singh and V. Venkatesh. 2013. Oropharyngeal candidiasis and Candida colonization in HIV positive patients in northern India. J. Infect. Dev. Ctries. 7(8): 608-613.

Merenstein D., H. Hu, C. Wang, P. Hamilton, M. Blackmon, H. Chen, R. Calderone and D. Li. 2013. Colonization by Candida species of the oral and vaginal mucosa in HIV-infected and noninfected women. AIDS Res. Hum. Retroviruses 29(1): 30-34.

Mitrovic S., V. Radovic, I. KranjcicZec, V. Arsic, A. Dzamic and D.J. Jevtovic. 1996. Oral candidiasis in HIV infected patients caused by Candida albicans and associated Candida species, Berkarda B. (eds). Monduzzi Editore, Bologna.

Nawrot U., M. Cisło, A. Noczyńska, K. Włodarczyk and E. Baran. 2006. Susceptibility to selected antifungal agents of yeast-like fungi 
isolated from gastrointestinal tract of diabetes children type 1. Mikol. Lek. 13(1): 35-38.

Ramírez M., M.C. Serrano, C. Castro, E. López, C. Almeida, A. Fernández, A. Romero and E. Martín-Mazuelos. 2006. Comparative study of disc diffusion and microdilution methods in susceptibility testing of micafungin against Candida species. J. Antimicrob. Chemother. 58(4): 861-863.

Romeo O. and G. Criseo. 2011. Candida africana and its closest relatives. Mycoses 54(6):475-86.

Sangeorzan J.A., S.F. Bradley, X. He, L.T. Zarins, G.L. Ridenour, R.N. Tiballi and C.A. Kauffman. 1994. Epidemiology of oral candidiasis in HIV-infected patients: colonization, infection, treatment, and emergence of fluconazole resistance. Am. J. Med. 97(4): 339-46. Sanitá P.V., E.G. de Oliveira Mima, A.C. Pavarina, J.H. Jorge, A.L. Machado and C.E. Vergani. 2013. Susceptibility profile of a Brazilian yeast stock collection of Candida species isolated from subjects with Candida-associated denture stomatitis with or without diabetes. Oral Surg. Oral Med. Oral Pathol. Oral Radiol. 116(5): 562-569.
Subcommittee on Antifungal Susceptibility Testing (AFST) of the ESCMID European Committee for Antimicrobial Susceptibility Testing (EUCAST). 2008. EUCAST definitive document EDef 7.1: method for the determination of broth dilution MICs of antifungal agents for fermentative yeasts. Clin. Microbiol. Infect. Off. Publ. Eur. Soc. Clin. Microbiol. Infect. Dis. 14(4): 398-405.

Subcommittee on Antifungal Susceptibility Testing (AFST) of the ESCMID European Committee for Antimicrobial Susceptibility Testing (EUCAST). 2014. Antifungal Agents. Breakpoint tables for interpretation of MICs. Version 7.0, valid from 2014-08-12. www. eucast.org.

Vargas K.G. and S. Joly. 2002. Carriage frequency, intensity of carriage, and strains of oral yeast species vary in the progression to oral candidiasis in human immunodeficiency virus-positive individuals. J. Clin. Microbiol. 40(2): 341-350.

Vergani C.E., P.V. Sanitá, E.G.O. Mima, A.C. Pavarina and A.L. Machado. 2013. Oral candidiasis: conventional and alternative treatment options. In: Candidiasis Epidemiol. Symptoms Treat. Options, pp. 85-115. 
